\title{
Cognitive therapy is more effective than fluoxetine in people with generalised social phobia
}

Clark DM, Ehlers A, McManus F, et al. Cognitive therapy versus fluoxetine in generalized social phobia: a randomized placebo-controlled trial. J Consult Clin Psychology 2003;71:1058-67.

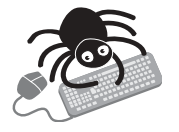

This article

contains extra text on the EBMH website

\section{What is the efficacy of cognitive therapy or fluoxetine in people with generalised social phobia?}

\section{METHODS}

$\square$

Design: Randomised placebo controlled trial.

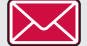

Allocation: Concealed for the two arms receiving medication.

Blinding: Double blind, for the two arms receiving medication.

Follow up period: Twelve months.

Setting: Institute of Psychiatry, London, UK; timeframe not stated.

Patients: Sixty people ( 31 female, 29 male) meeting DSM IV criteria for generalised social phobia, selected from 123 possible candidates. Mean age 33.2 years. Treatment groups similar with respect to marital and employment status, and education Exclusions: major depressive disorder, bipolar disorder, psychosis, epilepsy, borderline personality disorder, alcohol or substance dependence, previous treatment with SSRI, exposure therapy or cognitive therapy, refusal to withdraw from psychotropic medication 4 weeks before trial.

$\mathbf{R}_{\mathbf{C}}$

Intervention: For 16 weeks, three groups of 20 people received cognitive therapy (see www.ebmentalhealth.com/supplemental for further details), fluoxetine plus self-exposure, or placebo plus self-exposure. Sixteen sessions plus three booster sessions were offered.

Outcomes: Social phobia, measured with composite scale (based on 7 individual social phobia measures); general mood assessment (Beck Anxiety Inventory (BAl) and Beck Depression Inventory (BDI)) scales. Assessments completed by an independent assessor at $0,8,16,28$, and 52 weeks.

Patient follow up: $90 \%$ (six participants withdrew)

\section{MAIN RESULTS}

People in all three groups improved. At 16 weeks (post-treatment), and at 12 months follow up, cognitive therapy showed significant improvement in social phobia scales compared with fluoxetine plus self-exposure and placebo plus self-exposure (significance not stated; see http://www.ebmentalhealth.com/supplemental for table). There

For correspondence: David M Clark, Department of Psychology, Institute of Psychiatry, De Crespigny Park, London, UK; d.clark@iop.kcl.ac.uk Sources of funding: the Wellcome Trust. Eli Lilly provided the medication. were no significant differences between fluoxetine plus self-exposure and placebo plus self-exposure groups on social phobia scales. Compared with pretreatment assessments, mood scale scores improved, but there were few significant differences between groups.

\section{CONCLUSIONS}

Cognitive therapy more effective than fluoxetine or placebo for people with social phobia, although mood assessment scores were similar among groups.

\section{Commentary}

Ocial phobia is one of the most common anxiety disorders, with a lifetime prevalence ranging from $3 \%-13 \%$. "If untreated, the disorder tends to be chronic. In treatment settings, the most common form is generalised social phobia, in which the person fears and avoids a variety of social situations.

Several treatments have proven effective, particularly the various forms of cognitive-behaviour therapy (CBT) and the selective serotonin reuptake inhibitors (SSRIs), ${ }^{2}$ however they are far from universally effective, and there is ample room for improving outcome. Accordingly, the new CBT developed by David Clark and colleagues is of great interest (they call their treatment cognitive therapy, but as it contains exposure related exercises, it is more appropriately regarded as CBT). In this study, Clark and colleagues showed that their CBT is more effective than two other conditions (fluoxetine plus self-exposure, and pill placebo plus selfexposure) and that treatment gains were maintained at 12 months follow up. The effect sizes of Clark's CBT were greater than those previously published for other CBTs.

One strength of the Clark et al trial is that the investigators compared their CBT with fluoxetine combined with self-exposure. Combined SSRIs and exposure are commonly used in psychiatric practice, and so Clark et al's study examined one representative form of pharmacotherapy. It remains to be seen how their CBT compares with other drug regimens. It is not uncommon, for example, for psychiatrists to rotate their social phobia patients through different SSRIs or other drugs, and to augment SSRls with other agents. Future studies could compare CBT with these drug algorithms. A further important question is whether treatment outcome can be enhanced even further by combining CBT with pharmacotherapies.

Clark et al's outcome trial indicates that their new CBT shows great promise. But the findings are unlikely to have a great impact on clinical practice at the present time because of the lack of a readily available treatment manual. If such a manual becomes available, and if the initial results are independently replicated, then Clark's new CBT may have a profound impact on how we treat social phobia.

Steven Taylor, PhD

Professor, Department of Psychiatry, University of British Columbia, Vancouver, Canada

1 American Psychiatric Association. Diagnostic and statistical manual of mental disorders. Washington, DC: Author, 2000.

2 Fedoroff IC, Taylor S. Psychological and pharmacological treatments of social phobia: A meta-analysis. J Clin Psychopharm 2001;21:311-24. 